\title{
Analyse des pratiques d'embouche ovine dans deux zones semi-arides du Burkina Faso
}

\author{
Sanon Hadja Oumou ${ }^{1 *}$, Zorma Antoine ${ }^{2}$, Simian Aziz², Obulbiga M. Ferdinand ${ }^{1}$, Compaoré \\ Emmanuel ${ }^{3}$ \\ 1 INERA/Département Productions Animales, BP 910 Ouagadougou, Burkina Faso ; hadja osanon@yahoo.fr; Cel. \\ +22670102310 \\ 2 Institut du Développement Rural / Université Nazi Boni, 01 BP 1091 Bobo-Dioulasso 01 \\ 3 INERA/Département Gestion des Ressources Naturelles et Systèmes de Production, BP 910 Ouagadougou, \\ Burkina Faso
}

Original submitted in on $8^{\text {th }}$ April 2020. Published online at www.m.elewa.org/journals/ on $30^{\text {th }}$ June 2020 https://doi.org/10.35759/JABs.150.1

\begin{abstract}
RESUME
Objectif : Pour améliorer la productivité du bétail et de la viande et accroitre le revenu des producteurs, la promotion des activités d'embouche est encouragée et soutenu par différents acteurs au développement. Cependant, la maitrise de l'activité par les producteurs en milieu paysan est faiblement documentée.

Méthodologie et résultats : Cette étude a été conduite dans la zone semi-aride du Burkina Faso avec pour objectif d'analyser les pratiques d'embouche des producteurs et d'apprécier leur capacité de production. Elle a consisté en des enquêtes formelles auprès des emboucheurs de huit villages dont quatre dans la zone de Ouahigouya (province du Yatenga) et quatre dans la zone de Dori (province du Seno). Les données ont été collectées auprès de 197 producteurs choisis suivant un échantillonnage en boule de neige. Les résultats montrent deux groupes d'emboucheurs : le premier est dominé par les emboucheurs de la zone de Ouahigouya et le deuxième par ceux de la zone de Dori. Les emboucheurs du groupe 1 ont un âge plus avancé (54\% plus de 50 ans), que ceux du groupe 2 où près de $77 \%$ ont moins de 50 ans. Dans le premier groupe le niveau d'instruction est relativement plus élevé, avec $40 \%$ scolarisés contre 73 $\%$ d'illettrés pour le groupe 2. Le nombre d'ovins et de bovins embouchés est plus élevé au niveau du groupe 1 avec en moyenne 8,4 et 3,2 respectivement contre 3,8 et 2,3 pour le groupe 2. Les fosses fumières sont fréquentes dans le groupe 1 où $63 \%$ en possèdent.

Conclusion et application des résultats: En conclusion, l'étude montre un faible niveau technique des emboucheurs ainsi que leur faible capacité de production, plus marqué dans la zone de Dori. Ces résultats permettent de guider les interventions dans le domaine de l'embouche, notamment de cibler les actions suivant les sites. Dans ces démarches un accent particulier devra être mis sur les cultures à double objectif afin d'améliorer l'alimentation et réduire le cout de production, tout en favorisant l'intégration agricultureélevage.
\end{abstract}

Mots clés : Embouche, ovin, alimentation, capacité de production, Dori, Ouahigouya 


\section{ABSTRACT}

\section{Analysis of sheep fattening practices in two semiarid zones of Burkina Faso}

Objective: To improve animal and meat productivity and increase the revenue for farmers the promotion of fattening activities is encouraged and supported by many development actors. However, the knowledge of this activity by farmers in rural area is poorly documented.

Methodology and results: This study was conducted in the semi-arid zone of Burkina Faso in order to analyse the fattening practices of farmers and assess their production capacity. It consisted of formal surveys of farmers in eight villages, four of which were in the Ouahigouya area (Yatenga province) and four in the Dori area (Seno province). The data were collected from 197 farmers chosen according to a snowball sampling. The results show two groups of farmers: fatteners from the Ouahigouya area and the second dominate the first by those from the Dori area. The fatteners in group 1 are older ( $54 \%$ over 50 years) than those in group 2 where almost $77 \%$ are under 50 years old. In the first group, the level of education is relatively higher, with $40 \%$ educated against $73 \%$ illiterate for group 2 . The number of sheep and cattle fattened is higher in group 1 with on average 8.4 and 3.2 respectively against 3.8 and 2.3 for group 2 . Manure pits are frequent in group 1 where $63 \%$ have them.

Conclusion and application of results: This survey reveals low technical level of the farmers as well as their low production capacity, particularly in the Dori area. These results could guide interventions on fattening field particularly to target actions according to the sites. They should involve dual-purpose crop production in order to improve nutrition and reduce the cost of production, while promoting the integration of crop and livestock.

Key words: fattening, sheep, feeding, production capacity, Dori, Ouahigouya

\section{INTRODUCTION}

Au Burkina Faso, l'élevage constitue un maillon essentiel de l'économie nationale en contribuant pour plus de $18 \%$ au PIB et occupant la 3 ème place des produits d'exportation après l'or et le coton (FEWS NET, 2017). Cette activité occupe une frange importante de la population active et fournit des moyens d'existence à de nombreux ménages (82\% des ménages). En termes de sources de revenus monétaires pour les ruraux, il occupe la première place avec une contribution estimée à $38,8 \%$ et permet un accès facile aux services sociaux de base (MRA-PNUD, 2011). Ce soussecteur regorge de fortes potentialités dont un cheptel numériquement très important et diversifié avec 9,84 millions de bovins; 10,44 millions d'ovins; 15,63 millions de caprins ; 1,23 millions d'asins et plus de 37 millions de volaille (MRA, 2019). Ce qui fait du Burkina Faso un pays exportateur de bétail en direction de pays côtiers voisins (Ghana, Côte d'lvoire, Togo, Bénin). En 2014, les ovins ont occupé le premier rang des exportations avec 514000 têtes, suivis des caprins 401000 têtes et des bovins 344400 têtes (MRA,
2015). Cet élevage est à dominance traditionnelle extensive où les pâturages naturels constituent la principale source d'alimentation du bétail. II contribue à près de $85 \%$ dans l'alimentation des animaux (MRA, 2004), et est caractérisé par une faible productivité des animaux. Les systèmes intensifs ne concernent que $4,2 \%$ des bovins et $3,2 \%$ des petits ruminants (MRA-PNUD, 2011). La principale contrainte de l'élevage est essentiellement d'ordre alimentaire marqué entre autre par la faible productivité et la réduction des pâturages naturels, une faible valorisation des sous-produits agricoles, la faible pratique des cultures fourragères, la faible maîtrise des techniques de rationnement et la cherté des sousproduits agroindustriels (SPAl)que sont les tourteaux de coton, aliment composé, son cubé et mélasse. Pour améliorer la productivité et la compétitivité des filières animales, les autorités ont mis l'accent sur la liaison production et marché. Ainsi le Programme d'Appui aux Filières AgroSylvo-Pastorales (PAFASP) a été initié avec l'appui de la Banque Mondiale dans le but 
d'accroître la compétitivité des filières Agro-sylvopastorales sur les marchés nationaux et sousrégionaux. Pour ce qui concerne la filière bétailviande, ce PAFASP a entrepris des actions dont l'appui technique et matériel aux acteurs en amont afin d'accroître et améliorer la qualité de la production d'animaux de boucherie. Plusieurs projets et ONGs intervenant en milieu rural font également la promotion des activités d'embouche en apportant des appuis aux producteurs en vue de générer des revenus. Cependant, force est de reconnaitre que malgré ces efforts, la maitrise des bonnes pratiques techniques de cette activité par les producteurs en milieu réel n'est pas toujours satisfaisante, ce qui se traduit par de faibles gains de productivité. Pourtant, l'existence de marchés

\section{MATERIEL ET METHODES}

Sites d'étude: Dans la zone semi-aride du Burkina Faso, deux sites ont été retenus pour le déroulement de l'étude. Le département de Dori dans la province du Séno, et celui de Ouahigouya dans la province du Yatenga. Ces deux sites sont à vocation agropastorale, ont des effectifs de bétail relativement importants et des possibilités pour une meilleure intégration agricultureélevage. Ces deux zones appartiennent respectivement potentiels importants tant au niveau local (environ 25000 tonnes de viande) que sous régional à travers la structuration d'espaces d'échange sous régionaux dynamiques au niveau de l'UEMOA et de la CEDEAO (CRDI et ENDA DIAPOL, 2007), offre des opportunités de développement de ce secteur pour faire face à la demande croissante de produits animaux. Cette étude s'inscrit dans le cadre d'un projet d'intensification des productions agro-pastorales en vue d'améliorer les conditions de vie des producteurs (projet SIIL). Elle a été conduite dans la zone semi-aride du Burkina et a pour but de décrire les pratiques d'embouche bovine et ovine existantes, évaluer leur capacité de production, dégager les potentialités et contraintes y afférentes.

aux secteurs phytogéographiques sahéliens stricts et sub-sahélien (Fontès et Guinko, 1995). Plus précisément, quatre villages ont été concernés dans chaque zone. II s'agit des villages d'Aorèma, Bogoya, Tougou et Ziga dans la zone de Ouahigouya et de Bani, Gorgadji, MBamga et Seytenga dans la zone de Dori (Figure 1). 


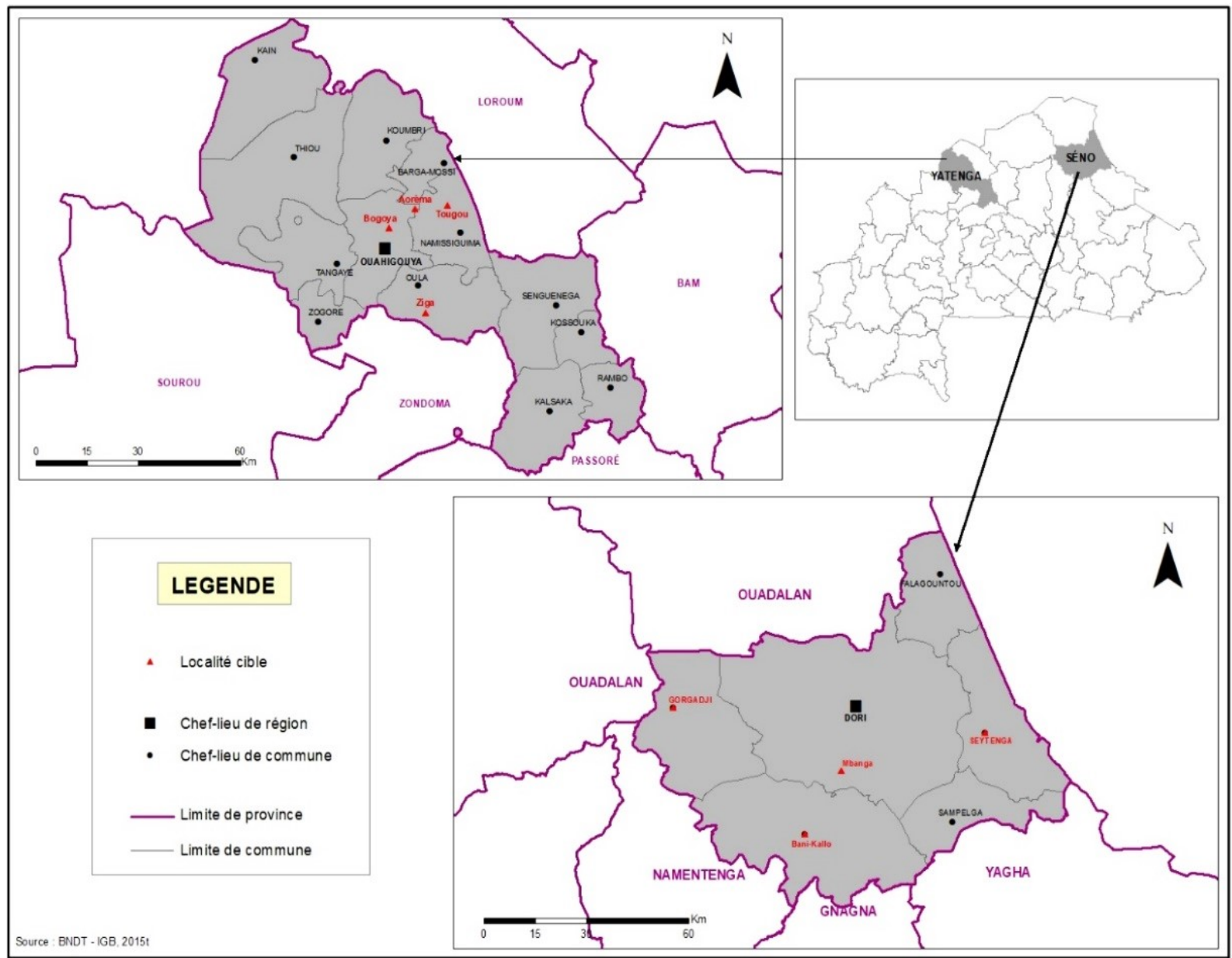

Figure 1. Localisation des sites d'étude

Le climat dans la zone de Ouahigouya est de type soudano-sahélien, caractérisé par une pluviométrie de 500 à $700 \mathrm{~mm}$ et une longue saison sèche de 7 à 9 mois ; on note une très grande variabilité interannuelle et une mauvaise répartition spatio-temporelle des pluies. De 2006 à 2016, le minimum d'eau tombée à Ouahigouya est de $505 \mathrm{~mm}$ en 2012 et le maximum de $983,4 \mathrm{~mm}$ en 2007. Au cours de la même période, le nombre de jours de pluies par saison agricole a varié de 48 à 71 jours respectivement. Les amplitudes thermiques sont très variables : les températures maximales atteignent $45^{\circ} \mathrm{C}$ (en avril) et les minimales $15^{\circ} \mathrm{C}$ (en février). La végétation dans la province du Yatenga comprend les principales formations végétales suivantes : - La brousse tigrée en îlot dispersés au nord ; - Les steppes, situées au nord de Ouahigouya ; - La savane arborée plus au sud dont l'étendue et la densité ont été réduites par les actions anthropiques. Les principales espèces sont : Khaya senegalensis, Tamarindus indica, Sclerocarya birrea dans les zones basses ; Parkia biglobosa, Acacia albida et Vitellaria paradoxum sur les champs les plus fertiles; les hauts de pente sont recouverts de formations très dégradées comportant des arbustes (Combretum micranthum, Guiera senegalensis), et une strate herbacée peu dense à base de Loudetia togoensis (Zorma, 2017). La population de la province était estimée à 553164 en 2006 , dont $52,77 \%$ de femmes, pour une population active (14-65 ans) de 285538 habitants, soit $52 \%$ de la population résidente (INSD, 2006). Les principales ethnies sont les Mossi $(85,6 \%)$, les Peulhs $(5,8 \%)$, les Samos (3,2\%) et les Marka (2,4\%) (Sawadogo, 2009). Les principales activités socioéconomiques sont l'agriculture, l'élevage et le commerce, auquel s'ajoutent l'artisanat et l'orpaillage. Dans la zone de Dori, le climat de type sahélien est caractérisé par une très faible pluviométrie comprise entre 400 et $600 \mathrm{~mm}$, une évaporation très intense; d'où une réserve en eau du sol très aléatoire. Les hauteurs d'eau recueillies entre 2010 et 2016 ont varié entre 432 et $623,9 \mathrm{~mm}$ 
avec un nombre moyen de jours de pluies entre 50 et 60 jours (Zorma, 2017). La végétation se présente sous la forme de peuplements ligneux ouverts avec des arbres espacés et dont les hauteurs dépassent très rarement sept mètres. C'est le domaine des steppes et les formations végétales rencontrées sont : - la steppe herbacée constituée par un tapis herbacé saisonnier, plus ou moins discontinu et parcouru par des zones de sols nus; - les steppes arborée et arbustive ; - les fourrés tigrés ou brousses tigrées constituées de touffes d'arbustes ou d'arbrisseaux souvent impénétrables à cause des épineux et - les formations rupicoles qui bordent les cours d'eau et les mares. Les principales espèces végétales rencontrées sont pour les ligneux divers types d'Acacia, Balanites aegyptiaca, Ziziphus mauritiana et au niveau des herbacées, Schoenefeldia gracilis, Aristida adcensionis, Zornia glochidata (Zoungrana (1991). La population de la province du Séno est estimée à 364080 habitants (en 2017) dont $50,30 \%$ de femmes. Cette population est essentiellement jeune car $57,09 \%$ de la population ont moins de 20 ans (INSD-DR/Sahel, 2017). Les principaux groupes ethniques présents sont les Peulh qui sont majoritaires, les Mossi, les Sonrhaï, les Gourmantché, les Djerma et les Bella. L'élevage constitue la principale activité socio-économique de cette population. L'agriculture pluviale dans la province est très peu favorable, la région enregistrant régulièrement des déficits importants.

Démarche méthodologique : Elle a consisté en des enquêtes formelles conduites auprès des agropasteurs dans les sites du projet intitulé « Intensification durable à travers une meilleure intégration des systèmes de production d'élevage et de cultures pour l'amélioration

\section{RESULTATS}

Typologie des pratiques d'embouche: La figure 2 montres la représentation des variables ayant servi à discriminer les groupes de producteurs. Sur l'axe 1, les variables suivantes sont discriminées : site, habitat, fosse fumière, activité principale, taille ménage, type d'embouche, niveau d'instruction, durée dans l'activité. Elles constituent des variables de structure des de la sécurité alimentaire et des avantages environnementaux dans la zone sahélienne du Burkina Faso ». Un questionnaire a été élaboré pour obtenir des informations sur les principaux points suivants afin de dégager les acquis et insuffisances de la production: - les caractéristiques socio-économiques des emboucheurs ; - les caractéristiques des ateliers ; les caractéristiques et paramètres de production ; - les ressources alimentaires. La population d'étude est constituée par des pratiquants d'embouche bovine et ovine dans les quatre villages sites dans chacune des deux zones soit huit villages. L'échantillonnage des producteurs a été réalisé selon la méthode « boule de neige ». Le point de départ est constitué par les producteurs cibles impliqués dans les activités du projet et pratiquant l'embouche; ces derniers ont été par la suite sollicités pour inclure d'autres producteurs pratiquant les activités d'embouche ovine et/ou bovine. Au total 100 producteurs ont été retenus dans la zone de Dori et 97 producteurs dans la zone de Ouahigouya. Les questionnaires ont été administrés aux chefs de ménage.

Analyses statistiques : L'analyse des données a été faite avec le programme SPSS version 22. Les données ont été soumises à une analyse des correspondances multiples, qui a permis d'obtenir une représentation des exploitations sous forme de projections sur les deux premiers axes factoriels. Des statistiques descriptives (fréquences, moyennes, variances, erreur-type, etc.) ont été effectuées sur les principales variables quantitatives caractéristiques des ateliers d'embouche. Le test de Khi 2 a permis de comparer les différents groupes de producteurs, avec un seuil de signification de $5 \%$.

exploitations. Sur l'axe 2, les variables, tourteaux de coton, fanes de légumineuses, pailles de céréales, pailles de brousse, prophylaxie, désinfection sont mieux représentées. Ces variables contribuent au fonctionnement de l'activité d'embouche, notamment l'alimentation et la santé des animaux. 


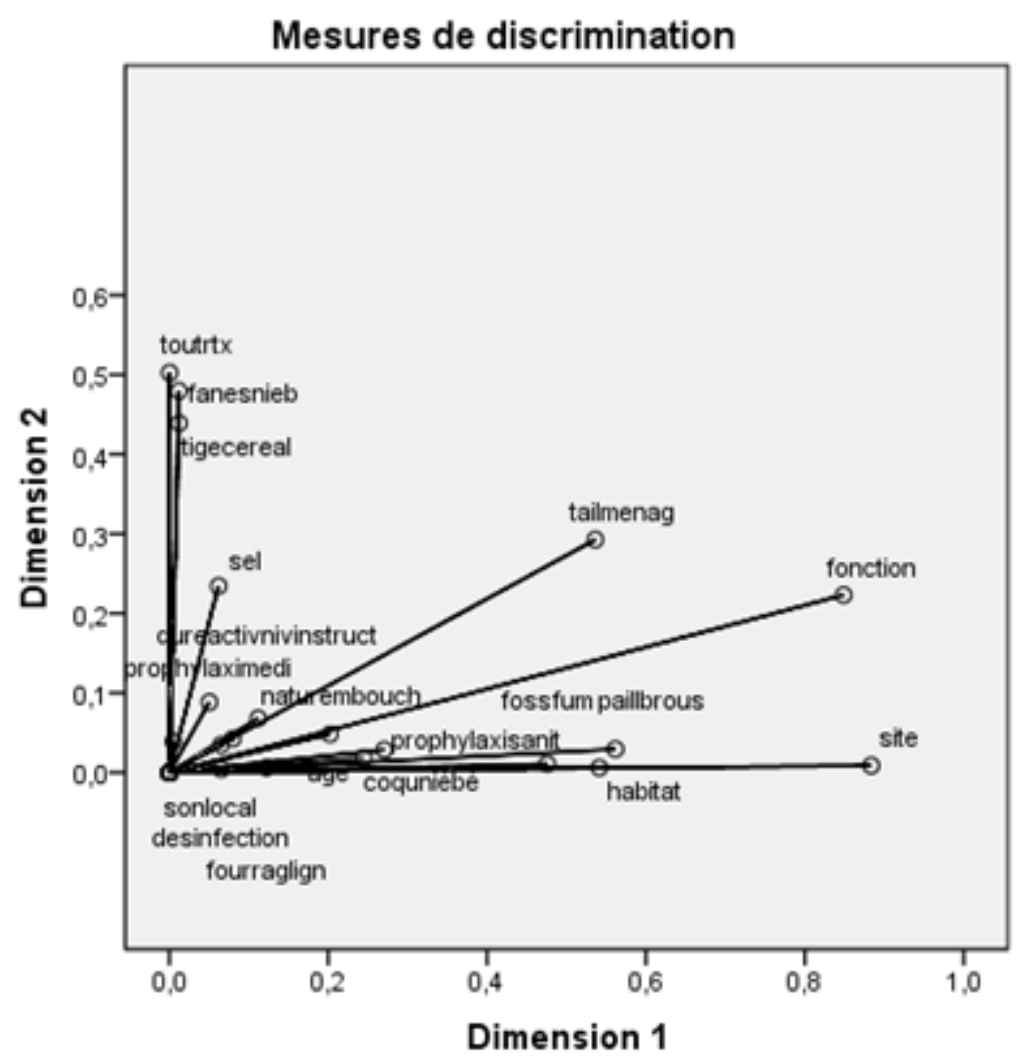

Figure 2 : Projection des variables dans le plan formé par les deux premières dimensions de l'Analyse des Composantes Multiples (ACM)

La figure 3 montre une discrimination des ateliers d'embouche en deux grands groupes. Le groupe 1 est constitué près de $78 \%$ d'ateliers d'embouche dans la zone d'Ouahigouya, tandis que le groupe 2 comprend majoritairement $91 \%$ d'ateliers d'embouche dans la zone de Dori. L'analyse statistique (test de Khi 2) montre une différence significative $(P \leq 0,05)$ entre les deux groupes d'emboucheurs par rapport aux paramètres suivants: le site, l'âge, le niveau d'instruction, la formation, la taille de ménage, la nature de l'habitat, la pratique de prophylaxie sanitaire, le nombre d'animaux par rotation et la présence de fosse fumière. 


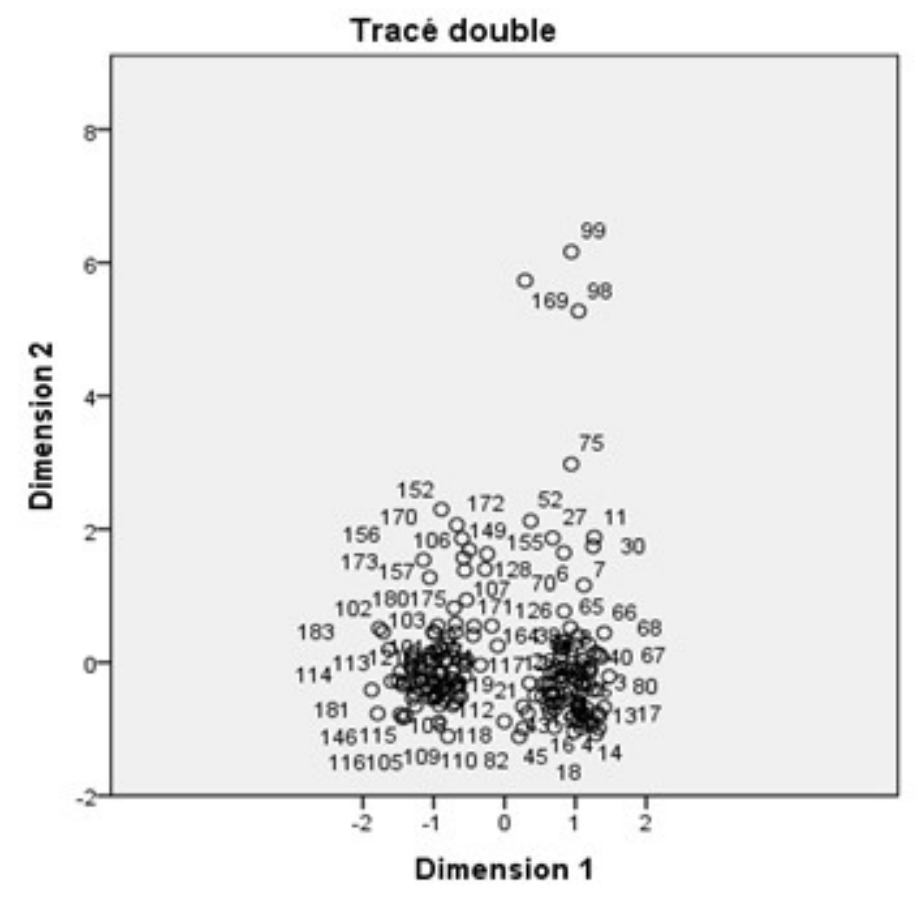

Normalisation variable principale.

Figure 3 : Projection des observations dans le plan formé par les deux premières dimensions de l'ACM

Caractéristiques des emboucheurs : Quelque fois la zone d'investigation, l'embouche est une activité plus pratiquée majoritairement par les hommes $(74,4 \%$ des enquêtés du groupe 1 et $80 \%$ du groupe 2) (tableau 1); la participation des femmes $(25,6 \%)$ est légèrement plus importante dans la zone de Dori. L'âge des pratiquants d'embouche diffère significativement suivant les 2 groupes. Les classes d'âges [40 à 60] ans dominent au niveau du groupe 1 $(58,6 \%)$ tandis que dans le groupe 2 ce sont les classes d'âges inférieures à 50 ans qui sont plus représentées $(76,8 \%)$. Ainsi, les emboucheurs de la zone de Dori sont plus jeunes comparés à ceux de la zone de Ouahigouya, où il y a aussi une proportion importante d'emboucheurs ayant plus de 60 ans $(25 \%)$. Les agropasteurs constituent la couche socioprofessionnelle qui s'investit le plus dans l'activité d'embouche dans la zone de Dori $(84,1 \%)$. Dans le groupe 1, ils sont en majorité des agriculteurs, ensuite viennent les agropasteurs. Concernant le niveau de l'instruction, la plupart des emboucheurs enquêtés sont illettrés avec une proportion plus importante dans le groupe $2(73,2 \%)$. Dans le groupe 1, un nombre appréciable d'emboucheurs sont scolarisés $(26,7 \%)$ ou ont fait l'école franco-arabe $(16,4 \%)$. Sur le plan organisationnel, $48,3 \%$ et $41,5 \%$ des emboucheurs des groupes 1 et 2 respectivement appartiennent à une organisation paysanne. Ils sont peu nombreux, les emboucheurs qui ont reçu un appui technique, matériel et financier. Les bénéficiaires d'une formation en embouche sont plus importants dans le groupe 1, environ $32 \%$ contre seulement $12 \%$ pour le groupe 2 . 
Tableau 1 : Caractéristiques générales des emboucheurs

\begin{tabular}{|c|c|c|c|c|}
\hline \multicolumn{2}{|l|}{ Paramètres } & Groupe 1 & Groupe 2 & P-value \\
\hline \multirow[t]{2}{*}{ Site } & Dori & 21,6 & 91,5 & \multirow[t]{2}{*}{$* * *$} \\
\hline & Ouahigouya & 78,4 & 08,5 & \\
\hline \multirow[t]{2}{*}{ Sexe } & Homme & 80,2 & 74,4 & \multirow[t]{2}{*}{ Ns } \\
\hline & Femme & 19,8 & 25,6 & \\
\hline \multirow[t]{4}{*}{ Age } & $<40$ & 16,4 & 34,1 & \multirow[t]{4}{*}{ 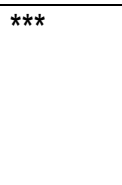 } \\
\hline & $40-50$ & 29,3 & 42,7 & \\
\hline & $50-60$ & 29,3 & 12,2 & \\
\hline & $>60$ & 25,0 & 11,0 & \\
\hline \multirow[t]{3}{*}{ Fonction } & Eleveur & 11,2 & 07,3 & \multirow[t]{3}{*}{ Ns } \\
\hline & Agriculteur & 57,8 & 08,5 & \\
\hline & agropasteur & 31,0 & 84,1 & \\
\hline \multirow[t]{4}{*}{ Niveau d'instruction } & Illettré & 50,9 & 73,2 & \multirow[t]{4}{*}{ ** } \\
\hline & alphabétisé & 06,0 & 03,7 & \\
\hline & Scolarisé & 26,7 & 15,9 & \\
\hline & franco-arabe & 16,4 & 07,3 & \\
\hline \multicolumn{2}{|c|}{ Appartenance à une OP } & 48,3 & 41,5 & Ns \\
\hline \multicolumn{2}{|c|}{ Bénéficiaire d'appui } & 27,6 & 30,5 & Ns \\
\hline \multicolumn{2}{|c|}{ Bénéficiaire de formation } & 31,9 & 12,2 & $* \star *$ \\
\hline
\end{tabular}

${ }^{* *}: P<0,001 ;{ }^{* *}: p<0,01 ;$ NS : non significatif

Caractéristiques des exploitations: II n'y a pas de différence significative entre les deux groupes d'embouche concernant le nombre d'année d'expérience dans l'activité, de même que la main d'œuvre utilisée. On note que les emboucheurs ayant moins de 10 ans d'expérience dominent dans les deux cas (environ $45 \%$ et $60 \%$ respectivement dans les groupes 1 et 2). Ceux ayant plus de 20 ans d'expérience sont plus rencontrés dans le groupe 1 (29
$\%)$ comparé au groupe 2 (16\%). La main d'œuvre mobilisée est essentiellement familiale dans les deux cas et la taille des ménages du groupe 1 (20 personnes) et significativement plus élevée que celle du groupe 2 (8 personnes). Le hangar sans clôture est utilisé comme principal habitat au niveau des ateliers d'embouche du groupe 2, tandis que pour le groupe 1, plus de la moitié des ateliers (52\%) utilise en plus des hangars, une clôture faite de grillage ou de banco.

Tableau 2. Caractérisation des exploitations

\begin{tabular}{|c|c|c|c|c|}
\hline - & & Groupe 1 & Groupe 2 & P-value \\
\hline \multirow{3}{*}{ Durée activité } & $<10$ ans & 44,83 & 59,76 & \multirow{3}{*}{ Ns } \\
\hline & $11-20$ & 25,86 & 24,39 & \\
\hline & $>20$ & 29,31 & 15,85 & \\
\hline \multirow{2}{*}{ Main d'œuvre } & Familiale & 95,69 & 100 & \multirow{2}{*}{ Ns } \\
\hline & Salariée & 03,45 & 0 & \\
\hline \multicolumn{2}{|l|}{ Taille ménage } & 20,36 & 8,43 & *** \\
\hline \multirow{3}{*}{ Nature habitat } & Hangar & 45,69 & 92,68 & \multirow{3}{*}{$* * *$} \\
\hline & hangar + clôture & 51,72 & 07,32 & \\
\hline & Bergerie & 02,59 & 0 & \\
\hline
\end{tabular}

${ }^{* * *}: \mathrm{P}<0,001 ; \mathrm{NS}:$ non significatif

Caractérisation de la production: Le tableau 3 montre les caractéristiques de la production des animaux d'embouche au niveau des deux groupes. Dans l'ensemble, peu de producteurs pratiquent l'embouche bovine uniquement (6 à $7 \%$ \%). Ceux pratiquant l'embouche ovine seule sont majoritaires dans le groupe 1 (53\%), suivi par ceux utilisant les deux espèces (bovin et ovin) (43\%). Dans le groupe 2 , ces derniers sont les plus nombreux (50\%). 
Au niveau de la santé, les mesures de prophylaxie médicales sont bien connues par les emboucheurs qui les pratiquent à plus de $95 \%$. Cependant, pour la prophylaxie sanitaire qui se résume à un lavage des animaux et du matériel d'élevage, les emboucheurs du groupe 2 sont nombreux à la pratiquer $(73 \%$, contre 45 $\%$ pour le groupe 1$)$. Les paramètres de production (nombre d'animaux embouchés, nombre de rotations, durée de l'opération, âge des animaux) varient en fonction de la nature de l'embouche et des groupes. Le nombre d'ovins et de bovins embouchés par rotation est significativement plus élevé pour le groupe 1 comparé au groupe 2 avec respectivement 8,4 ovins et
3,8 bovins contre 3,2 ovins et 2,3 bovins. L'âge des animaux à l'entrée en embouche ne diffère pas suivant les groupes: on note en moyenne 1 an (13 à 14 mois) pour les ovins et 4 ans pour les bovins. La durée par opération d'embouche ovine comme bovine est similaire pour les deux groupes. Elle est en moyenne de 6 mois pour les ovins et 5 mois pour les bovins. L'aménagement de fosses fumière pour le stockage des déjections en vue de son utilisation en agriculture est plus connu au niveau du groupe 1 où environ $63 \%$ des emboucheurs en disposent, contre seulement 13\% de ceux du groupe 2 .

Tableau 3 : Caractérisation et paramètres de production

\begin{tabular}{|c|c|c|c|c|}
\hline \multicolumn{2}{|c|}{ Paramètres } & Groupe 1 & Groupe 2 & P-value \\
\hline \multirow{3}{*}{ Nature de l'embouche } & Bovine & 06,03 & 07,32 & \multirow{3}{*}{ Ns } \\
\hline & Ovine & 53,45 & 42,68 & \\
\hline & bovine et ovine & 40,52 & 50,00 & \\
\hline \multicolumn{2}{|l|}{ Prophylaxie médicale } & 95,69 & 96,34 & Ns \\
\hline \multicolumn{2}{|l|}{ Prophylaxie sanitaire } & 44,83 & 73,17 & $* * \star$ \\
\hline \multicolumn{2}{|c|}{ Nombre d'ovins par rotation } & 8,4 & 3,2 & $* \star \star$ \\
\hline \multicolumn{2}{|c|}{ Nombre de bovins par rotation } & 3,8 & 2,3 & ** \\
\hline \multicolumn{2}{|c|}{ Age des ovins d'embouche (mois) } & 13,3 & 14,7 & Ns \\
\hline \multicolumn{2}{|c|}{ Age des bovins d'embouche (an) } & 3,95 & 3,65 & Ns \\
\hline \multicolumn{2}{|c|}{ Durée de l'opération / ovins (mois) } & 6,28 & 5,87 & Ns \\
\hline \multicolumn{2}{|c|}{ Durée de l'opération / bovins (mois) } & 4,78 & 5,29 & Ns \\
\hline \multicolumn{2}{|c|}{ Nombre de rotation par an } & 1,33 & 1,17 & * \\
\hline \multicolumn{2}{|c|}{ Présence de fosse fumière } & 62,93 & 13,41 & $\star * \star *$ \\
\hline
\end{tabular}

${ }^{* * *}: P<0,001 ;{ }^{* *}: p<0,01 ; p<0,05 ;$ NS : non significatif

Ressources alimentaires : Divers types de ressources alimentaires sont utilisés pour les animaux d'embouche. Pour chaque type, les proportions des emboucheurs varient suivant les deux groupes, sauf pour les pailles de céréales et les fanes de légumineuses qui constituent les aliments de base et systématiquement utilisés par presque tous les emboucheurs. Les proportions des emboucheurs utilisant les foins/pailles de brousse, les fourrages ligneux, les tourteaux de coton et le son local diffèrent significativement entre les deux groupes. Les emboucheurs utilisant les fourrages ligneux (gousses de Acacia sp. de Piliostigma reticulatum) et de coques de niébé sont importants dans le groupe 1 (13 et 26\% contre 4 et $5 \%$ dans le groupe 2). L'utilisation de foin ou paille de brousse est plus pratiquée par les emboucheurs du groupe $2(90 \%)$; ces derniers sont également nombreux à utiliser les tourteaux de coton et le son local dans l'alimentation des animaux d'embouche (98 et $85 \%$ respectivement contre 90 et $71 \%$ au niveau du groupe 1 ). 
Tableau 4 : Ressources alimentaires utilisées en embouche

\begin{tabular}{|l|l|l|l|}
\hline & Groupe 1 & Groupe 2 & p-value \\
\hline Foin / paille de pâturage & 37,07 & 90,24 & $\star \star *$ \\
\hline Paille de céréales & 96,55 & 97,56 & Ns \\
\hline Fanes de légumineuses & 95,69 & 96,34 & Ns \\
\hline Fourrage ligneux & 12,93 & 03,66 & $*$ \\
\hline Coques de niébé & 25,86 & 04,88 & $\star \star *$ \\
\hline Tourteaux de coton & 89,66 & 97,56 & $*$ \\
\hline Son local & 71,55 & 85,37 & $*$ \\
\hline Sel & 86,21 & 86,59 & Ns \\
\hline
\end{tabular}

${ }^{* * *}: P<0,001 ; * *: p<0,01 ; p<0,05 ;$ NS : non significatif

\section{DISCUSSION}

L'activité d'embouche est essentiellement pratiquée par les hommes dans les deux groupes. La faible implication des femmes à l'activité d'embouche est en accord avec les résultats rapportés par Sanon et al. (2014) qui ont noté un taux de $23,8 \%$ de femmes pratiquant l'embouche dans l'ouest du Burkina Faso. Cette situation pourrait s'expliquer par le fait que les hommes sont généralement les propriétaires des animaux, dans la mesure où nous avons constaté que les femmes intervenaient dans les taches d'alimentation et d'entretien des animaux. La proportion importante des emboucheurs ayant un âge de plus de 50 ans dans le groupe 1, pourrait indiquer que les jeunes dans ce groupe s'intéressent moins aux activités d'embouche. En effet, ce groupe est constitué en majorité des emboucheurs de la zone de Ouahigouya où les jeunes s'adonnent plus à l'orpaillage. Notre résultat pour le groupe 2, avec près de $77 \%$ des producteurs âgés de moins de 50 ans corrobore celui rapporté par Sanon et al. (2014) dans l'ouest du Burkina où $67 \%$ à $78 \%$ des emboucheurs avaient moins de 50 ans. Ce résultat montre que l'activité d'embouche est principalement pratiquée par des acteurs matures et tant à devenir une profession permanente pour ces acteurs. La forte implication des agriculteurs et des agropasteurs dans l'activité d'embouche montre que cette activité est un moyen important de l'intégration agriculture - élevage. En effet, elle permet de valoriser les résidus de culture et de disposer des fumiers qui servent à fertiliser les champs. Sur le plan instruction, le taux important (plus de 50\%) des emboucheurs non scolarisés ou alphabétisés peut constituer un handicap pour l'adoption des technologies améliorées de production, et même pour l'enregistrement des opérations au cours de l'activité, afin de déterminer les coûts de production. Ainsi, nous avons noté qu'aucun producteur ne dispose de cahier de suivi et ne peut évaluer de façon raisonnable les dépenses engagées. II s'en suit une impossibilité d'évaluer la rentabilité de l'activité, même si les producteurs affirment y gagner assez de revenu. Gufa et al. (2017) ont noté $65 \%$ des répondants illettrés dans une étude sur les systèmes d'embouche en Ethiopie qui est similaire à nos résultats. La faible affiliation à une organisation paysanne, ne favorise pas l'obtention d'appui quelconque. En effet, ces deux paramètres sont généralement liés car les ONG et projets s'appuient sur les OP pour apporter leur aide. Les résultats d'appartenance à une organisation sont inférieurs à celui obtenu par Sanon et al. (2014) à l'Ouest du Burkina Faso $(57,67 \%)$. Cependant, la proportion relativement élevée (32\%) des emboucheurs du groupe 1 , ayant bénéficié d'une formation pourrait s'expliquer par la présence de projets/ONG œuvrant pour le renforcement des capacités techniques des producteurs dans cette zone. La prépondérance des emboucheurs avec moins de 10 ans d'expérience dans l'activité pourrait indiquer que les producteurs s'intéressent de plus en plus à l'embouche pour générer des revenus. Cependant, la main d'œuvre demeure majoritairement familiale dans les deux groupes, ce qui pose les difficultés d'évaluation des charges réelles de l'activité. L'habitat sommaire (hangar avec ou sans enclos) chez la majorité des emboucheurs des deux groupes montre qu'il y a peu d'investissement. Ce type d'habitat bien que réduisant l'effet de la chaleur et assurant une protection contre les vols d'animaux fréquents dans la zone, limite l'application des mesures d'hygiène, car laissant pénétrer l'eau des pluies. Seulement 2,6\% des exploitations dans le groupe 1 disposent d'une bergerie. Nos résultats sont inférieurs à ceux rapportés par Drabo (2011) qui a noté $48,61 \%$ d'étables et bergeries modernes en matériaux définitifs (parpaing, pierres taillées et banco amélioré) dans l'Ouest du Burkina Faso. Nos résultats montrent la dominance de 
l'embouche ovine et une combinaison des deux espèces (ovine et bovine) par rapport à l'embouche exclusivement bovine (6\%). Ce qui indiquerait l'importance des ovins sur le plan social dans cette zone du Burkina Faso comme rapporté par Boly et al. (2001). En effet, les ovins constituent la première espèce utilisée comme sacrifice lors de la fête de Tabaski. Les mesures prophylactiques, surtout celle médicale, bien connues par les emboucheurs sont en accord avec les résultats rapportés par Sanon et al. (2014) dans l'Ouest du Burkina Faso, qui a relevé que tous les emboucheurs pratiquaient la prophylaxie médicale, tandis que la prophylaxie sanitaire était faiblement appliquée. Ces auteurs ont noté $40 \%$ de producteurs appliquant la prophylaxie sanitaire au niveau des ateliers semi-intensifs, ce qui se rapproche de nos résultats dans le groupe $1(45 \%)$. Cette attitude montre que les emboucheurs sont conscients de la nécessité de maintenir les animaux en bonne santé dans l'atelier d'embouche. La prophylaxie sanitaire se résume au balayage des locaux et le lavage des ovins et des matériels pour l'alimentation (mangeoire, abreuvoir). Des efforts restent à faire pour améliorer l'hygiène des locaux, des animaux et des aliments. Le nombre moyen d'ovins au niveau du groupe $1(8,4)$ est similaire à celui rapporté par Sanon et Traoré (2014) pour les ovins en embouche semi intensive dans l'Ouest du Burkina Faso. Pour les bovins, les résultats $(2,3$ à 3,8$)$ sont nettement inférieurs à ceux obtenus par Sanon et al. (2014) qui ont noté de 7 à 16, voir 50 bovins en embouche semi intensive et intensive. La durée de l'activité d'embouche des ovins de 5 à 6 mois est supérieure à la durée moyenne au niveau national qui est d'environ 4 mois (110 à 120 jours) (MRA, 2005) et de celle (115 jours) notée par Yacouba et al. (2004) au Mali. Elle est cependant inférieure à 7 mois rapporté par Lucol (2003) à Dosso au Niger. Cette durée d'embouche relativement longue pourrait s'expliquer par l'âge d'entrée des animaux en embouche qui est plus bas. Cela pourrait s'expliquer aussi par une méconnaissance et une faible technicité des producteurs. Au vu de ces résultats, l'embouche pratiquée dans les deux zones serait de type semi intensif compte tenu du nombre d'animaux réduit et de la durée observée, tel que relevé par INERA/DPA (2011). On peut donc dire que la capacité des emboucheurs de la zone d'étude est faible. Pour la zone de Dori, réputé pour ses potentialités pastorales avec des effectifs important de cheptel $(15,72 \%$ des petits ruminants et $20,55 \%$ de bovins du pays en 2018) (MRAH, 2019), ce résultat suggèrerait que les éleveurs s'intéressent peu à l'embouche, préférant l'exploitation des animaux provenant d'élevage naisseur. Ce qui montre le caractère social de l'élevage plutôt qu'économique. Une étude comparative des coûts de production des ovins de ces deux provenances et en dehors de la période de Tabaski serait intéressante afin de faire des recommandations propices. Par contre l'aspect économique serait plus important dans la zone de Ouahigouya. L'aménagement de fosses fumière en atelier d'embouche est une pratique encouragée, permettant une exploitation judicieuse du fumier qui est utilisé pour fertiliser les champs. La proportion élevée d'emboucheurs disposant de fosses fumières dans le groupe 1, montre que ce groupe accorde une importance particulière à la production des cultures ; ils sont plus à vocation agricole comparé au groupe 2 . Cette pratique leur permet de renforcer l'intégration agriculture élevage et de tirer le maximum de profit en termes de production des cultures et des performances animales suite à l'utilisation des résidus de culture. Cependant, la faible utilisation de fosses fumières dans le groupe 2 (principalement la zone de Dori), pourrait s'expliquer par certaine réticence des producteurs face à cette innovation due selon eux, aux difficultés de maintenir les fosses sur des sols qui sont sableux pour la plupart sans un grand investissement. Le principe de combiner les trois types de ressources alimentaires (aliments grossiers, concentrés et minéraux) bien connu par l'ensemble des producteurs est important pour la réussite de l'opération d'embouche. La faible utilisation des foins/pailles de brousse par les emboucheurs du groupe 1, essentiellement de la zone de Ouahigouya, pourrait s'expliquer par la faible disponibilité de cette ressource dans cette zone. Ainsi, les producteurs se tournent vers d'autres types d'aliments tels que les gousses de fourrages ligneux (Piliostigma reticulatum) et les coques de niébé qui sont beaucoup utilisés dans cette zone. Ce qui confirme l'importance et la place des aliments non conventionnels dans la pratique de l'embouche en milieu paysan comme souligner par beaucoup d'auteurs (Fall-Touré et al., 1997 ; Zoundi et al., 2003 et Kiema et al., 2008). Ainsi, moins de producteurs du groupe 1 utilisent les tourteaux de coton et le son local qui sont les aliments concentrés couramment utilisés. Le recours aux ressources alimentaires locales permet ainsi de réduire le coût de l'alimentation des animaux d'embouche (Sanon et al., 2014). La quasi permanence des résidus de culture dans l'alimentation des animaux d'embouche, montre la nécessité d'améliorer la valeur alimentaire de ces fourrages. Un moyen simple est 
l'utilisation des variétés de culture à double objectif qui ont l'avantage de garder les fourrages verts à la maturité des graines et ainsi des valeurs nutritives plus élevées comparées à celles des variétés locales, aussi

\section{CONCLUSION}

Cette étude a permis de comprendre les réalités inhérentes de la pratique de l'activité d'embouche dans la région sahélienne du Burkina Faso. Elle a mis en exergue les acquis et les insuffisances de cette activité. Les emboucheurs sont pour la plupart des agropasteurs, pouvant ainsi stocker des résidus de culture pour l'alimentation des animaux en stabulation. Ils ont cependant une faible capacité de production et un faible niveau technique, plus marqué au niveau des emboucheurs de la zone de Dori. Cette zone étant constituée en majorité de pasteurs dont l'activité principale est l'élevage extensif, la sensibilisation et la formation doivent être maintenues, afin de permettre

\section{REMERCIEMENTS}

Cette étude a été réalisé dans le cadre du projet "Sustainable intensification through better integration of crop and livestock production systems for improved food security and environmental benefits in Sahelian

\section{REFERENCES BIBLIOGRAPHIQUES}

Boly $H$, llboudo JB, Ouédraogo $M$, Berti $F$, Lebally $P$, Leroy P. 2001. L'élevage du "mouton de case" : aspects techniques, socio-économiques et perspectives d'amélioration au Yatenga (Burkina Faso). Biotechnol. Agron. Soc. Environ., 5(4) : 201-208

CRDI et ENDA DIAPOL, 2007. Les dynamiques transfrontalières en Afrique de l'Ouest. Paris/Karthala ; 219p/

Drabo A, 2011. Diagnostic des pratiques d'embouche bovine et ovine dans l'ouest du Burkina Faso. Mémoire de fin d'études, option Vulgarisation, Institut du Développement Rural, Université Polytechnique de Bobo-Dioulasso, $106 \mathrm{p}$.

Fall-Touré S, Traoré E, N'Diaye K, N'Dèye SN, Sèye BM, 1997. Utilisation des fruits de Feidherbia albida pour l'alimentation des bovins d'embouche paysanne dans le bassin arachidier au Sénégal. Livestock Research for Rural Development. 9 (5): 19p.

FEWS NET (Famine Early Warning Systems Network), 2017. Les fondamentaux du marché des denrées de base et du bétail au Burkina Faso. Ouagadougou, 47p. bien de céréales que de légumineuses (Zorma, 2017 ; Simian 2017). Ces variétés contribuent ainsi à réduire le coût d'alimentation des animaux et favorisent l'intégration agriculture - élevage.

leur engagement dans le système plus intensif (embouche) permettant l'acquisition de quantité importante de fumier et de revenus pouvant servir entre autre à l'achat des aliments pour maintenir le reste du troupeau en période de soudure. Dans la zone de Ouahigouya, où le contexte est plus favorable avec l'agropastoralisme pratiqué et le niveau appréciable des producteurs. L'accompagnement est nécessaire pour améliorer davantage leur productivité. Tous les acteurs de la chaine sont alors interpellés pour le renforcement des capacités techniques des producteurs et une meilleure organisation de l'activité d'embouche qui est un moyen sûr de lutte contre la pauvreté en milieu rural.

zone of Burkina Faso», financé par le Laboratoire d'Innovation pour I'Intensification Durable (SIIL) du programme Feed the Future (FtF) de l'USAID, auquel les auteurs adressent leurs vifs remerciements.

Fontes J. and Guinko S, 1995. Carte de la végétation et de l'occupation du sol du Burkina Faso. Note explicative. Ministère de la Coopération Française, Toulouse, France, 67 pp.

Gumayde Gufa, Ayelech Sana, Taju Hussein, 2017. Assessment of Sheep Husbandry and Fattening System in Daramillo Woreda Gamo Gofa Zone, Southern Ethiopia. Food Science and Quality Management ; Vol. 60 : 48-54.

INERA/DPA (Institut de l'Environnement et de Recherches Agricoles/Département Productions Animales), 2013. Référentiels Technico-économiques (RTE) sur l'embouche ovine au Burkina Faso, version finale, Ouagadougou, Burkina Faso, 56 p. + annexes.

INSD/DR Sahel, 2017. Recueil statistique de la région du Sahel, Burkina Faso

INSD, 2006. Recueil statistique de la Région du Nord, Burkina Faso. 129 p.

Kiéma A, Nianogo AJ, Ouédraogo T, Somda J, 2008. Valorisation des ressources alimentaires locales dans l'embouche ovine paysanne: performances technico-économiques et 
options de diffusion. Rev. Cahiers Agricultures Vol.17, $n^{\circ} 1$, Janvier-février 2008, p. 23-27.

Lucol Fabienne, 2003. L'embouche - ovine et bovine en milieu paysan à Dosso (Niger) : aspects technico-économiques et perspectives d'amélioration. Montpellier : UM2, 54 p. Mémoire DESS: Productions animales en régions chaudes : Université Montpellier 2.

MRA (Ministère des Ressources Animales), 2005. Initiative Elevage, Pauvreté et Croissance (IEPC): descriptif détaillé des systèmes d'élevage modélisé, Ouagadougou, non paginé.

MRA (Ministère des Ressources Animales), 2015. Annuaires des statistiques de l'élevage 2014. Ouagadougou, 177p.

MRAH (Ministère des Ressources Animales et Halieutiques), 2019. Annuaires des statistiques de l'élevage. Ouagadougou, 140p.

MRA-PNUD (Ministère des Ressources Animales Programme des Unis pour le Développement), 2011. Contribution de l'élevage à l'économie et à la lutte contre la pauvreté ; les déterminants de son développement. Ouagadougou, 88p.

Sanon HO, Traoret L, 2014. Typologie d'ateliers d'embouche ovine au Burkina Faso (Ouest) et performances des unités pilotes. Livestock Research for Rural Development 26 (10) 2014. $9 p$.

Sanon HO, Drabo A, Sangaré M, Kiendrebeogo T, Gomgnibou A, 2014. Caractérisation des pratiques d'embouches bovine et ovine dans l'Ouest du Burkina Faso. Int. J. Biol. Chem. Sci. 8(2) : 536-550, April 2014. http://ajol. Info/index.php/ijbcs.

Sawadogo M. 2009, Plan Communal de Développement de Ouahigouya. Ouahigouya, $152 p$.

Simian A, 2017. Production et valeur alimentaire des résidus de culture des variétés à double objectifs utilisés dans l'alimentation animale dans la province du Yatenga. Mémoire de fin de cycle, option Elevage, Institut du Développement Rural, Université Nazi Boni, Burkina Faso, 62 p.

Yacouba S, Hassoumi G, Hamani D, 2004. Analyse technique et économique de l'embouche bovine et ovine dans trois zones agroécologiques du Niger. Rapport final d'étude. Dir. Etudes Program. Resour. Anim. Niger.
Serv. Coop. d'Action Cult. L'Ambassade Fr Niamey, Niger, 96p.

Zorma A, 2017. Production et valeur alimentaire des résidus de culture de variétés à double objectifs utilisés dans l'alimentation animale dans la région sahélienne du Burkina Faso. Mémoire de fin de cycle, option Elevage, Institut du Développement Rural, Université Nazi Boni, Burkina Faso, 65 p.

Zoundi SJ, Sawadogo LL, Nianogo JA, 2003. Pratiques et stratégies paysannes en matière de complémentation des ruminants au sein des systèmes d'exploitation mixte agricultureélevage du plateau central et du nord du Burkina Faso. Tropicultura, 21: 122-128.

Zoungrana I., 1991. Recherche sur les aires pâturées du Burkina Faso. Thèse doctorat Sciences Naturelles, Université Bordeaux III. UFR Aménagement et Ressources naturelles. 277p. 